\title{
UNA NUEVA ESPECIE DEL GÉNERO PRIONOSPIO MALMGREN, 1867 (ANNELIDA, SPIONIDAE) PROCEDENTE DEL SURESTE DEL GOLFO DE VIZCAYA
}

\author{
Julián Martínez ${ }^{1}$ \& Idoia Adarraga ${ }^{2}$ \\ 1,2 Sociedad Cultural INSUB. Museo Okendo. Apdo. 3223, 20080 Donostia-San Sebastián, Spain. \\ Email: julidoia@outlook.com - ${ }^{1}$ ORCID iD: https://orcid.org/0000-0002-4043-241X - ${ }^{2}$ ORCID iD: \\ https://orcid.org/0000-0002-9600-8212
}

\section{RESUMEN}

Una nueva especie, Prionospio rikardoi n. sp. ha sido identificada en fondos circalitorales de las costas del País Vasco (SE del golfo de Vizcaya). La nueva especie se caracteriza por poseer crestas dorsales en varios segmentos corporales y cuatro pares de branquias lisas, apinnadas y relativamente cortas en los setígeros $2-5$. En el presente artículo se describe, ilustra y compara la nueva especie con especies próximas. Además, se aporta una clave de identificación de todas las especies válidas del género Prionospio Malmgren, 1867 con sólo branquias apinnadas que comienzan en el segundo setígero. El material tipo ha sido depositado en el Museo Nacional de Ciencias Naturales de Madrid.

$$
\text { urn:Isid:zoobank.org:pub:6F3B8DDB-1E72-47E6-8454-08E7B68524E0 }
$$

Palabras clave: Annelida; Spionidae; Prionospio; nueva especie; golfo de Vizcaya.

\section{ABSTRACT}

A new species of the genus Prionospio Malmgren, 1867 (Annelida, Spionidae) from SE of Bay of Biscay

A new species, Prionospio rikardoi n. sp. has been identified from circalittoral soft bottoms of Basque coast (SE Bay of Biscay). The new species is characterized by having dorsal crests in several of their body segments as well as four pairs of smooth, apinnate branchiae on chaetigers 2-5. In the present paper, the new species is described, illustrated and compared to related species. In addition, and identification key is provided for all know valid Prionospio Malmgren, 1867 species possessing only apinnate branchiae beginning on chaetiger 2. The Material Type has been deposited in the Museo Nacional de Ciencias Naturales of Madrid.

Keywords: Annelida; Spionidae; Prionospio; new species; Bay of Biscay.

Recibido/Received: 16/05/2019; Aceptado/Accepted: 4/09/2019; Publicado en línea/Published online: 02/10/2019

Cómo citar este artículo/Citation: Martínez, J. \& Adarraga, I. 2019. Una nueva especie del género Prionospio Malmgren, 1867 (Annelida, Spionidae) procedente del sureste del golfo de Vizcaya. Graellsia, 75(2): e099. https://doi.org/10.3989/ graellsia.2019.v75.244

Copyright: (c) 2019 SAM \& CSIC. This is an open-access article distributed under the terms of the Creative Commons Attribution 4.0 International (CC BY 4.0) License. 


\section{Introducción}

Dentro de los anélidos poliquetos, Prionospio constituye probablemente el complejo específico más heterogéneo y diverso de la familia Spionidae (Radashevsky, 2015). Hasta siete géneros (Prionospio Malmgren, 1867; Streblospio Webster, 1879; Paraprionospio Caullery, 1914; Apoprionospio Foster, 1969; Orthoprionospio Blake \& Kudenov, 1978; Aurospio Maciolek, 1981a y Laubieriellus Maciolek, 1981b) han sido involucrados en este complejo (Zhou \& Li, 2009). A su vez, dentro del género Prionospio (sensu lato), algunos autores como Blake \& Kudenov (1978) y Maciolek (1985) distinguen tres subgéneros en función del tipo de branquias: Prionospio Malmgren (sensu stricto) donde incluyen especies con branquias apinnadas y pinnadas simultáneamente, Minuspio Foster, 1971 cuyas especies poseen únicamente branquias apinnadas y Aquilospio Foster, 1971 donde las especies portan únicamente branquias pinnadas.

Aunque esta última división subgenérica se considera actualmente artificial y sin valor taxonómico (Wilson, 1990, Sigvaldadóttir, 1998; Radashevsky, 2015), ha sido adoptada por diversos autores (Imajima, 1990; Blake, 1996; Dagli \& Çinar, 2009, 2011) para diferenciar morfotipos de Prionospio.

Más recientemente, otras discrepancias taxonómicas han surgido en torno a los criterios empleados para la definición de los géneros Prionospio y Aurospio (Paterson et al., 2016; Blake et al., 2017; Peixoto \& Paiva, 2019). Autores como Blake et al. (2017) sugieren que ciertas especies atribuidas al género Aurospio por Sigvaldadóttir (2002), Mincks et al. (2009) y Paterson et al. (2016) deberían incluirse en realidad dentro del género Prionospio.

A día de hoy, alrededor de 125 especies nominales de Prionospio han sido citadas en todo el mundo, de las cuales unas 90 se consideran especies válidas (Read \& Fauchald, 2019).

En el transcurso de una revisión de ejemplares de la familia Spionidae recolectados en diversas campañas oceanográficas efectuadas en las costas del País Vasco (SE del golfo de Vizcaya), se han identificado 35 ejemplares de una especie desconocida del género Prionospio portadora únicamente de branquias apinnadas en los setígeros 2-5.

En el presente artículo se describe e ilustra dicha especie, a la vez que se aporta una clave taxonómica de todas las especies válidas de Prionospio con únicamente branquias apinnadas comenzando en el segundo setígero.

\section{Material y métodos}

\section{ÁREA DE ESTUDIO}

Todos los ejemplares proceden de fondos blandos circalitorales de la plataforma continental de las costas de Gipuzkoa y Bizkaia (País Vasco, SE del golfo de Vizcaya), a profundidades comprendidas entre $36 \mathrm{y}$ $105 \mathrm{~m}$ (Fig. 1).

\section{ANÁLISIS Y RECOLECCIÓN DE LAS MUESTRAS}

Las muestras fueron recogidas en el transcurso de varias campañas oceanográficas llevadas a cabo entre los años 2003 y 2014 por AZTI-TECNALIA. En cada una de las estaciones se obtuvieron tres réplicas bentónicas con una draga Smith-McIntyre de 0,1 $\mathrm{m}^{2}$ de superficie efectiva. El material así obtenido se hizo pasar a través de un tamiz de $1 \mathrm{~mm}$ de luz de malla para la selección de la macrofauna. La fracción retenida se introdujo en recipientes de plástico, fijando las muestras en formaldehido al $4 \%$ en agua de mar, neutralizadas con bórax. En el laboratorio se tiñeron las muestras con rosa de bengala durante un periodo

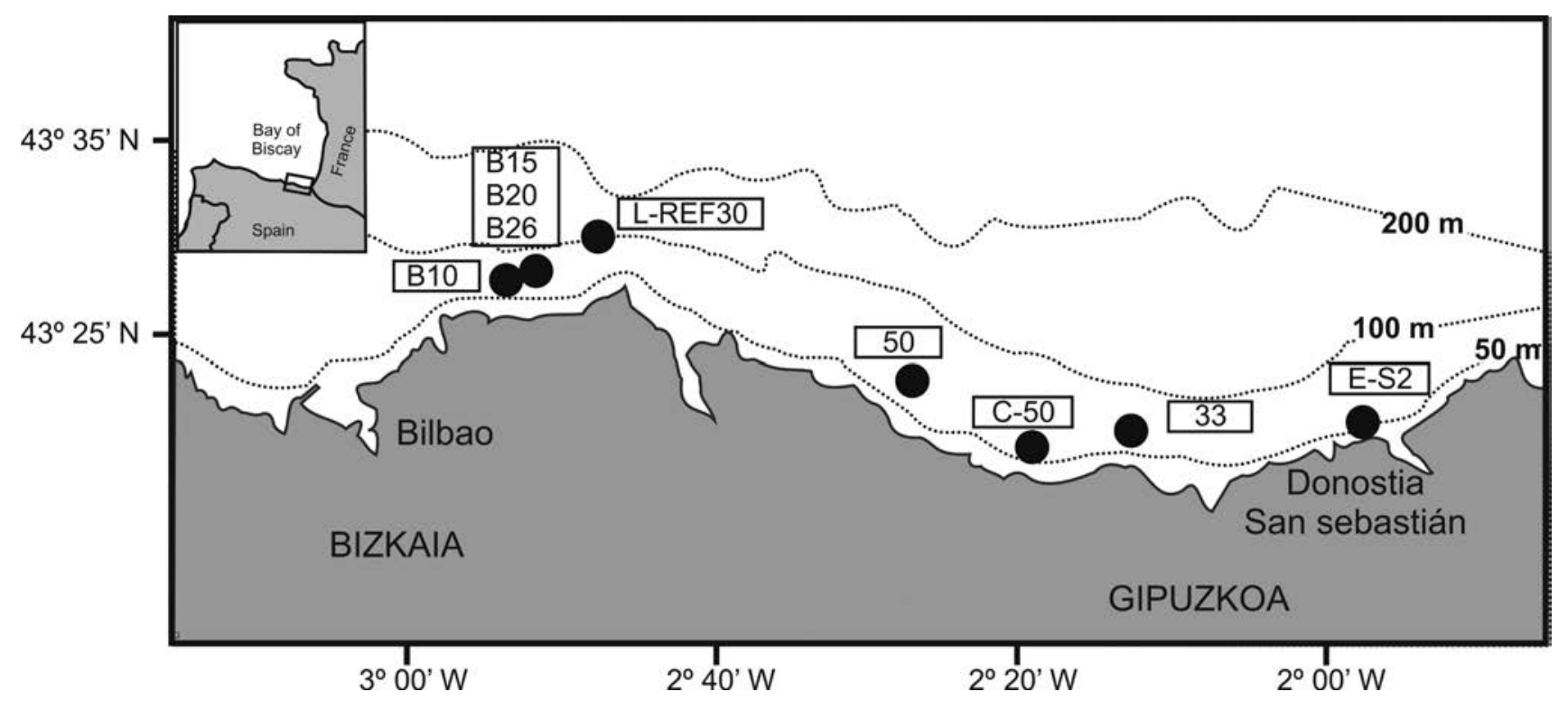

Fig. 1.- Área de estudio y localización de las estaciones de muestreo.

Fig. 1.- Study area and locations of sampling stations. 
de $24 \mathrm{~h}$. A continuación se procedió a la separación e identificación taxonómica, utilizándose lupas binoculares y microscopios ópticos. Todos los ejemplares obtenidos fueron conservados en etanol al $70 \%$. Las fotografías de los ejemplares se realizaron con la ayuda de una cámara fotográfica Nikon D7200 acoplada a un microscopio Zeiss. Para las mediciones se emplearon una cámara clara y un ocular micrométrico adaptados al microscopio.

En cada estación, previa a la filtración, se destinó una porción del sedimento para su caracterización físico-química. Los análisis granulométricos se realizaron mediante el uso de una columna de 8 tamices $(4 \mathrm{~mm}$ a $31 \mu \mathrm{m})$. Los porcentajes de gravas, arenas y fango fueron calculados como fracción $>2 \mathrm{~mm}, 63 \mu \mathrm{m}$ a 2 $\mathrm{mm}$ y $<63 \mu \mathrm{m}$, respectivamente (Holme \& McIntyre 1971 en Borja et al., 2006).

El material tipo de la nueva especie Prionospio rikardoi n. sp. ha sido depositado en la colección del Museo Nacional de Ciencias Naturales de Madrid, España.

Para la designación de todas las especies de Prionospio incluidas en el artículo se ha seguido el criterio adoptado en el Registro Mundial de Especies Marinas (WoRMS) (Read \& Fauchald, 2019).

\section{Resultados}

Phylum Annelida

Familia Spionidae Grube, 1850

Genus Prionospio Malmgren, 1867 sensu stricto

\section{Prionospio rikardoi $\mathrm{n} . \mathrm{sp}$.}

u r n: 1s id: z o o b a n k. or g: a c t: D 085 D 6BB-63E6-4780-ABBA-01CE0D1576F1

Figs. 2-5

\section{MATERIAL TIPO}

Holotipo. MNCN 16.01/18526, ejemplar incompleto (región anterior de 14,91 $\mathrm{mm}$ de longitud y $0,72 \mathrm{~mm}$ de anchura), Armintza, Bizkaia, estación $\mathrm{B} 10,43^{\circ} 27.439^{\prime} \mathrm{N}, 02^{\circ} 53.594^{\prime} \mathrm{W}$, arenas gruesas, $59 \mathrm{~m}$ de profundidad, 05.10.2011.

Paratipos. MNCN 16.01/18527, 5 ejemplares (fragmentos anteriores), Deba, Gipuzkoa, estación C50, 43 $19.5^{\prime} \mathrm{N}, 02^{\circ} 20^{\prime} \mathrm{W}$, arenas, $53 \mathrm{~m}$, 06.03.2003; MNCN 16.01/18528, 6 ejemplares (fragmentos anteriores), Deba, Gipuzkoa, estación C50, $43^{\circ} 19.5^{\prime} \mathrm{N}, 02^{\circ} 20^{\prime} \mathrm{W}$, arenas, $53 \mathrm{~m}, 22.06 .2004$; MNCN 16.01/18529, 1 ejemplar (fragmento anterior), Donostia-San Sebastián, Gipuzkoa, esta-

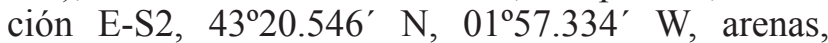
36 m, 27.08.2007; MNCN 16.01/18530, 1 ejemplar (fragmento anterior), Lekeitio, Bizkaia, estación $50,43^{\circ} 23.487^{\prime} \mathrm{N}, 02^{\circ} 27.944^{\prime} \mathrm{W}$, gravas, $71 \mathrm{~m}$, 02.06.2009; MNCN 16.01/18531, 1 ejemplar (fragmento anterior), Getaria, Gipuzkoa, estación 33, $43^{\circ} 19.987^{\prime} \mathrm{N}, 02^{\circ} 12.571^{\prime} \mathrm{W}$, arenas gruesas, $67 \mathrm{~m}$,
03.06.2009; MNCN 16.01/18532, 3 ejemplares (fragmentos anteriores), Armintza, Bizkaia, estación B10, $43^{\circ} 27.439^{\prime} \mathrm{N}, 02^{\circ} 53.594^{\prime} \mathrm{W}$, arenas gruesas, $59 \mathrm{~m}$, 05.10.2011; MNCN 16.01/18533, 9 ejemplares (fragmentos anteriores, incluidas 4 regiones posteriores), Armintza, Bizkaia, estación B15, 43⒉923' N, $02^{\circ} 52.794^{\prime} \mathrm{W}$, arenas, $74 \mathrm{~m}, 05.10 .2011$; MNCN 16.01/18534, 2 ejemplares (fragmentos anteriores, incluida 1 región posterior), Armintza, Bizkaia, estación $\mathrm{B} 20,43^{\circ} 28.366^{\prime} \mathrm{N}, 02^{\circ} 53.069^{\prime} \mathrm{W}$, gravas, $85 \mathrm{~m}$, 05.10.2011; MNCN 16.01/18535, 3 ejemplares (fragmentos anteriores), Armintza, Bizkaia, estación B26, $43^{\circ} 28.470^{\prime} \mathrm{N}, 02^{\circ} 52.061^{\prime} \mathrm{W}$, arenas gruesas, $87 \mathrm{~m}$, 05.10.2011; MNCN 16.01/18536, 1 ejemplar (fragmento anterior), Armintza, Bizkaia, estación B26, $43^{\circ} 28.470^{\prime} \mathrm{N}, 02^{\circ} 52.061^{\prime} \mathrm{W}$, arenas gruesas, $87 \mathrm{~m}$, 06.05.2014; MNCN 16.01/18537, 2 ejemplares (fragmentos anteriores, incluida 1 región posterior), Bakio, Bizkaia, estación L-REF30, 43³0.001' N, 02²47.994' $\mathrm{W}$, arenas gruesas, $105 \mathrm{~m}, 06.03 .2013$.

\section{DESCRIPCIÓN}

Todos los ejemplares incompletos. Holotipo consistente en una región anterior de $14,91 \mathrm{~mm}$ de largo con 57 setígeros y $0,72 \mathrm{~mm}$ de ancho. Paratipos comprendiendo regiones anteriores de 2,62-15,05 $\mathrm{mm}$ de largo, $11-47$ setígeros y $0,39-0,76 \mathrm{~mm}$ de ancho; y regiones posteriores con 3,31-8,98 mm de largo, 15-32 setígeros y $0,26-0,52 \mathrm{~mm}$ de ancho.

Cuerpo alargado, delgado, subcilíndrico (Figs. 2A, 4A). Color en alcohol blanco a beige claro. Prostomio en forma de botella invertida, ligeramente truncado anteriormente, sin protuberancias marginales (Fig. 2A); carúncula estrecha extendiéndose hasta el margen posterior del setígero 2 (Figs. 2A, 4B). Un par de órganos nucales en forma de $\mathrm{V}$ a ambos lados de la carúncula (Fig. 4B). Dos pares de ojos subdérmicos rojizos a marrones dispuestos trapezoidalmente; par anterior pequeño, esférico, par posterior notablemente mayor, en forma de media luna (Figs. 2B, 5A). Palpos ausentes en todos los especímenes. Peristomio rodeando el prostomio, parcialmente fusionado con el setígero 1, formando moderadas alas laterales (Figs. 2A-B, 4C).

Cuatro pares de branquias cilíndricas, apinnadas, marginalmente ciliadas, presentes en los setígeros 2-5; primer par aproximadamente 1,3-2 veces más largo que el resto; segundo, tercer y cuarto par de longitud similar (Figs. 2A-B, 4A, D).

Lamelas notopodiales postsetales del setígero 1 fusionadas a las caras dorsales posteriores del peristomio, redondeadas a subtriangulares (Figs. 3A, 4B). Lamelas notopodiales de los setígeros 2-6 esbeltas, triangulares, erectas, mayores en los setígeros 3-5 (Figs. 2A-B, 3B-F); cambiando a cuadrangulares redondeadas en los siguientes (Figs. 2A, 3G-H); ovales a triangulares y más agudas en los últimos setígeros (Fig. 3I). Entre los setígeros 7-22/30 las lamelas notopodiales 

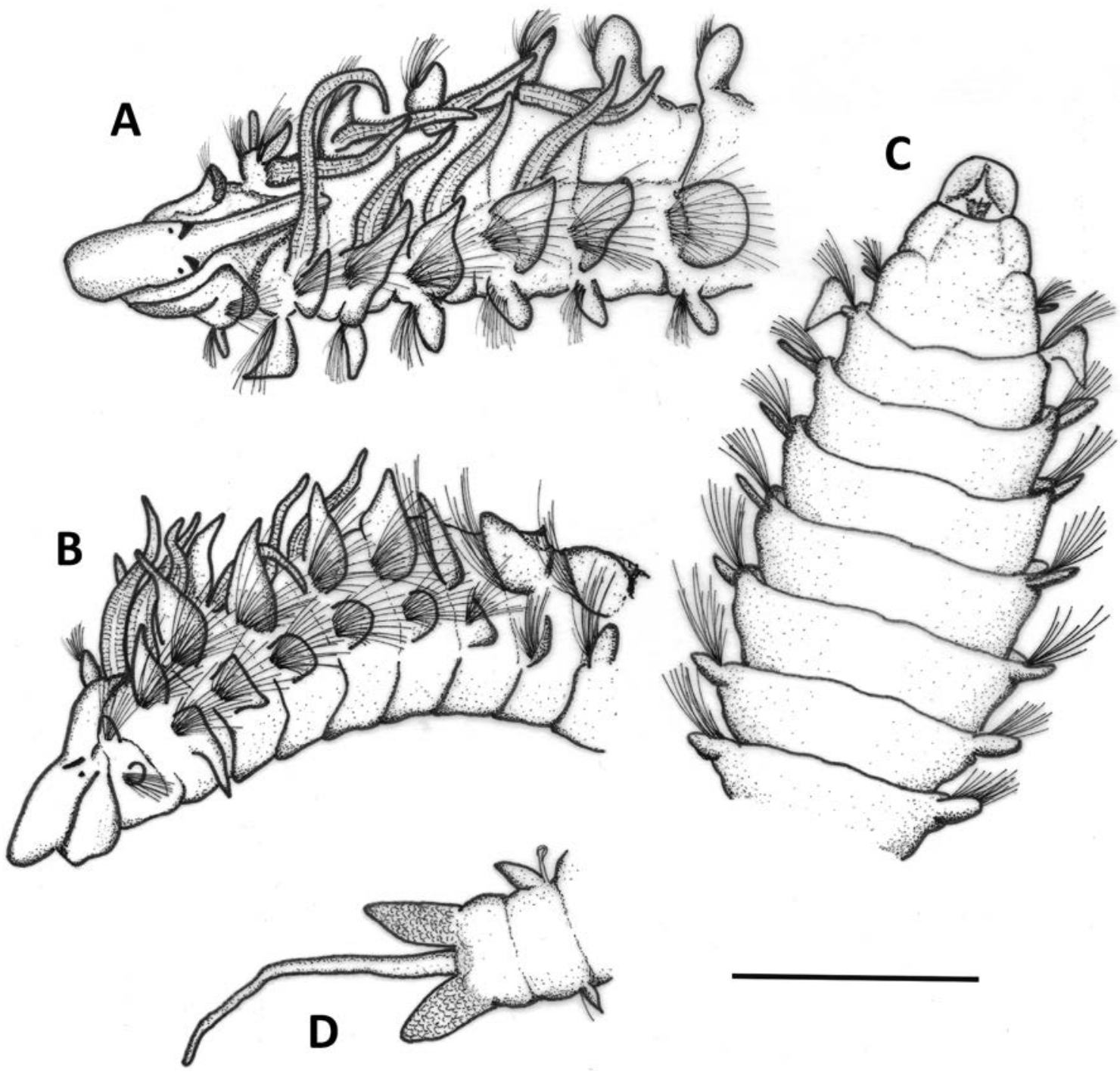

Fig. 2.- Prionospio rikardoi n. sp. A. Región anterior, vista dorsolateral (paratipo MNCN 16.01/18535). B. Región anterior, vista lateroventral (paratipo MNCN 16.01/18528). C. Región anterior, vista ventral (paratipo MNCN 16.01/18527). D. Pigidio, vista ventral (paratipo MNCN 16.01/18534). Escalas: A: $500 \mu \mathrm{m}$; B: $600 \mu \mathrm{m}$; C: $750 \mu \mathrm{m}$; D: $340 \mu \mathrm{m}$.

Fig. 2.- Prionospio rikardoi n. sp. A. Anterior end, dorsolateral view (paratype MNCN 16.01/18535). B. Anterior end, lateroventral view (paratype MNCN 16.01/18528). C. Anterior end, ventral view (paratype MNCN 16.01/18527). D. Pygidium ventral view (paratype MNCN 16.01/18534). Scale bar: A: $500 \mu \mathrm{m}$; B: $600 \mu \mathrm{m}$; C: $750 \mu \mathrm{m}$; D: $340 \mu \mathrm{m}$.

se unen dorsalmente formando crestas dorsales bien definidas (Fig. 5A-C). Lamelas notopodiales presetales inconspicuas en el setígero 1 , redondeadas en los siguientes setígeros, disminuyendo su grado de desarrollo hacia el final del cuerpo (Fig. 3A-I).

Lamelas neuropodiales postsetales del setígero 1 redondeadas (Figs. 3A, 4E); acuminadas y alargadas ventralmente en el setígero 2 (Figs. 3B, 4E); trapezoidales, más agudas ventralmente en el setígero 3 (Figs. 3C, 4E); ovales a redondeadas a partir del cuarto setígero (Fig. 2B, 3D-H); más agudas y triangulares en los últimos setígeros (Fig 3I). Lamelas presetales neuropodiales redondeadas. Márgenes ventrales de los setígeros 8-19/26 sobresaliendo anteriormente, algunos de manera conspicua, pero sin llegar a definir auténticas crestas ventrales (Figs. 2C, 5D). Bolsas interparapodiales ausentes.
Noto- y neuropodios de los setígeros anteriores con sedas capilares largas, limbadas, granuladas, dispuestas en dos filas, más largas las de la fila posterior y con el limbo más estrecho (Figs. 3J-K, 5E-F). A partir de los setígeros 15-25 las sedas capilares se disponen en una única fila, son más delgadas y el limbo y granulación superficial desaparece progresivamente; Sedas en sable ventrales granuladas, presentes desde el setígero 10 hasta el final del cuerpo, en número de 1-2 por rama; robustas en los setígeros anteriores, más curvadas, largas, con un filamento distal (Fig. 3L) en el resto del cuerpo. Ganchos encapuchados ventrales comenzando a partir del setígero 15-20, en número máximo de 10 por fascículo, portando 4-5 pares de dientes secundarios por encima del diente principal; capuchón secundario presente, aparente en algunos ganchos pero inconspicuo en 


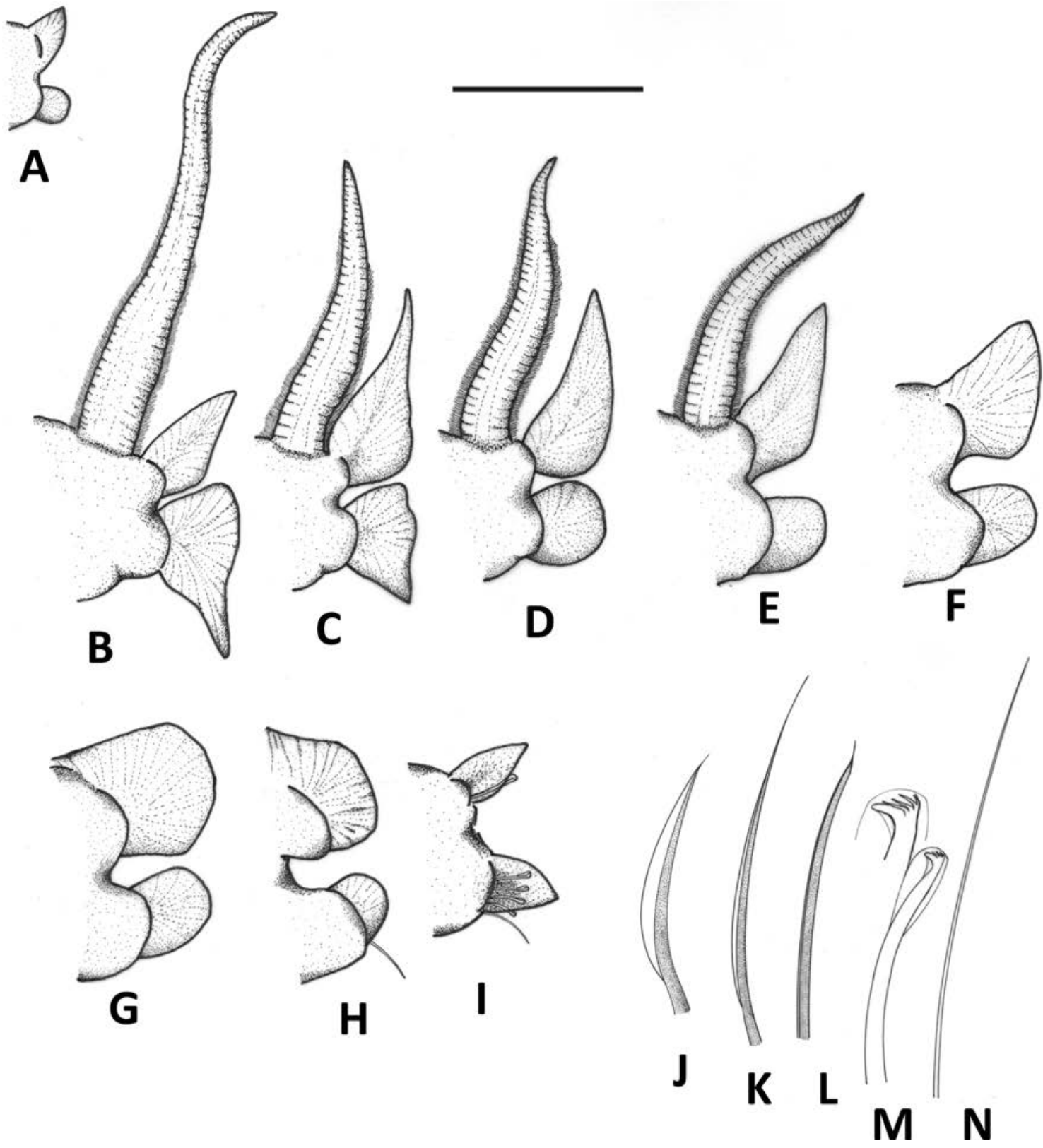

Fig. 3.- Prionospio rikardoi n. sp. A-H. Parápodos de los setígeros 1, 2, 3, 4, 5, 6, 8, 16, vista anterior (paratipo MNCN 16.01/18527). I. Setígero de la región final del cuerpo, vista anterior (paratipo MNCN 16.01/18532) (sedas capilares excluidas). J-N. Paratipo MNCN 16.01/18527. J. Seda capilar notopodial de la fila anterior de un setígero anterior. K Seda capilar notopodial de la fila posterior de un setígero anterior. L. Seda en sable de un setígero medio. M. Gancho encapuchonado neuropodial de un

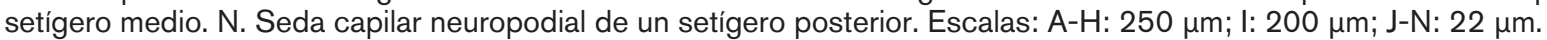

Fig. 3.- Prionospio rikardoi n. sp. A-H. Parapodia of chaetigers 1, 2, 3, 4, 5, 6, 8, 16, anterior view (paratype MNCN 16.01/18527). I. Posterior chaetiger, anterior view (paratype MNCN 16.01/18532) (capillary chaetae excluded). J-N. Paratype MNCN 16.01/18527. J. Notopodial capillary chaeta from anterior row of anterior chaetigers. K. Notopodial capillary chaeta from posterior row of anterior chaetigers. L. Sabre chaeta from middle chaetigers. M. Neuropodial hooded hook from middle

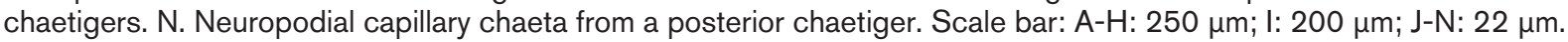

otros (Figs. 3M, 5G). Ganchos notopodiales encapuchados a partir del setígero $37-44$, hasta 8 por fascículo y de morfología similar a los neuropodiales. Sedas capilares lisas sin limbo en los setígeros medios y posteriores acompañando a los ganchos en ambas ramas (Fig. 3N).

Pigidio con tres cirros, uno más largo dorsomedial y dos más cortos ventrolaterales (Fig. 2D).

\section{OBSERVACIONES}

Prionospio rikardoi n. sp. se caracteriza fundamentalmente por poseer cuatro pares de branquias apinnadas entre los setígeros 2-5. Este mismo número y carácter de las branquias ha sido encontrado en las especies: Prionospio elegantula Imajima, 1990, Prionospio fauchaldi Maciolek, 1985, Prionospio japonicus Okuda, 1935, Prionospio laciniosa 

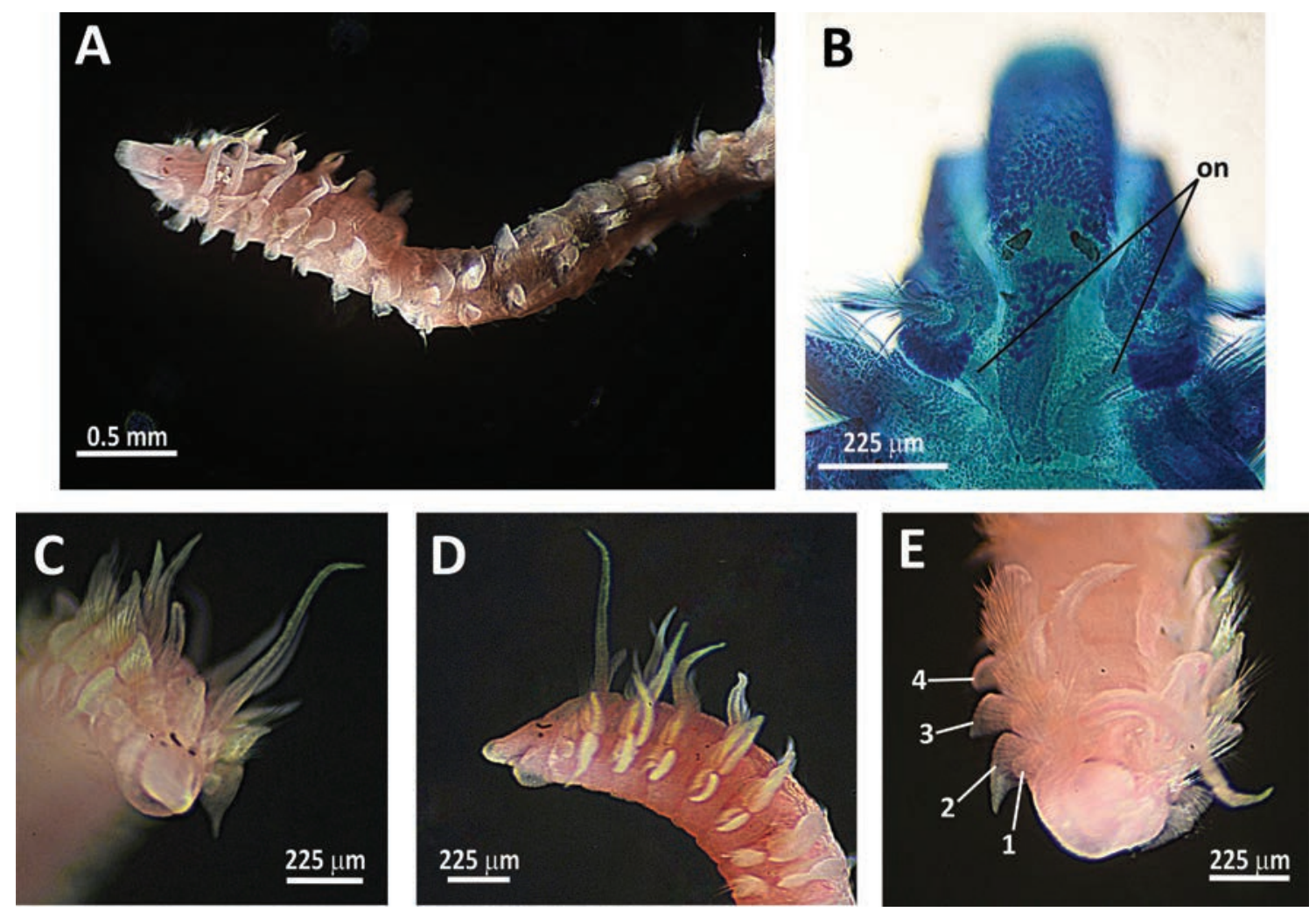

Fig. 4.- Prionospio rikardoi n. sp. A. Región anterior, vista dorsolateral (paratipo MNCN 16.01/18535). B. Prostomio, vista dorsal (paratipo MNCN 16.01/18528). C. Región anterior, vista frontolateral (paratipo MNCN 16.01/18533). D. Región anterior, vista lateral (paratipo MNCN 16.01/18533). E. Región anterior, detalle de los lóbulos neuropodiales postsetales de los setígeros 1-4 (flechas) (paratipo MNCN 16.01/18532). Abreviatura: on: órgano nucal.

Fig. 4.- Prionospio rikardoi n. sp. A. Anterior end, dorsolateral view (paratype MNCN 16.01/18535). B. Prostomium, dorsal view (paratype MNCN 16.01/18528). C. Anterior end, front lateral view (paratype MNCN 16.01/18533). D. Anterior end, lateral view (paratype MNCN 16.01/18533). E. Anterior end, dorsofrontal view, detail of neuropodial postchaetal lobes of chaetigers 1-4 (arrows) (paratype MNCN 16.01/18532). Abbreviation: on: nuchal organ.

Maciolek, 1985 y Prionospio vallensis Neal \& Paterson in Paterson et al., 2016.

De todas ellas, Prionospio rikardoi n. sp. se asemeja más a $P$. japonicus, especie originaria de las costas de Japón (Okuda, 1935). Ambas especies poseen cuatro pares de branquias cirriformes, lisas y densamente ciliadas, de las cuales el primer par es claramente más largo que el resto. Entre los rasgos que permiten diferenciarlas destacan la morfología del prostomio (triangular en P. japonicus, en forma de botella en Prionospio rikardoi n. sp.), extensión de la carúncula (hasta el margen posterior del setígero 1 en $P$. japonicus, margen posterior del setígero 2 en Prionospio rikardoi $\mathbf{n}$. sp.), aspecto de los ojos (dos pares de ojos esféricos en P. japonicus, par anterior esférico y par posterior en forma de media luna en Prionospio rikardoi n. sp.), forma de la lamela postsetal neuropodial del setígero 2 (cuadrangular en $P$. japonicus, acuminada y alargada ventralmente en Prionospio rikardoi n. sp.), presencia/ausencia de crestas dorsales (ausentes en P. japonicus, presentes en Prionospio rikardoi n. sp.) y longitud del cirro dorsomedial del pigidio (corto en P. japonicus, largo en Prionospio rikardoi n. sp.).
Prionospio rikardoi $\mathbf{n}$. sp. se distingue inmediatamente de $P$. elegantula por la longitud de sus branquias (relativamente cortas y con el par anterior más largo en Prionospio rikardoi n. sp., extremadamente largas y de longitud similar en P. elegantula). Otras diferencias serían la forma del prostomio (forma de botella en Prionospio rikardoi n. sp., triangular en P. elegantula), extensión de la carúncula (hasta el margen posterior del setígero 2 en Prionospio rikardoi $\mathbf{n}$. sp., margen posterior del setígero 1 en $P$. elegantula), forma de los ojos (par anterior esférico y par posterior en forma de media luna en Prionospio rikardoi n. sp., los dos pares de ojos esféricos en $P$. elegantula) y la forma de las lamelas postsetales neuropodiales de los setígeros 2 y 3 (acuminadas y trapezoidales respectivamente en Prionospio rikardoi n. sp., redondeadas ambas en P. elegantula).

Respecto a $P$. fauchaldi, $P$. laciniosa y $P$. vallensis, Prionospio rikardoi n. sp. se diferencia por poseer todas sus branquias completamente lisas; mientras que en las otras tres especies, algunas o todas ellas presentan la superficie arrugada y plegada ("wrinkled"). Complementariamente, la nueva especie se distingue por la forma de las sedas en sable (robustas en Prionospio 

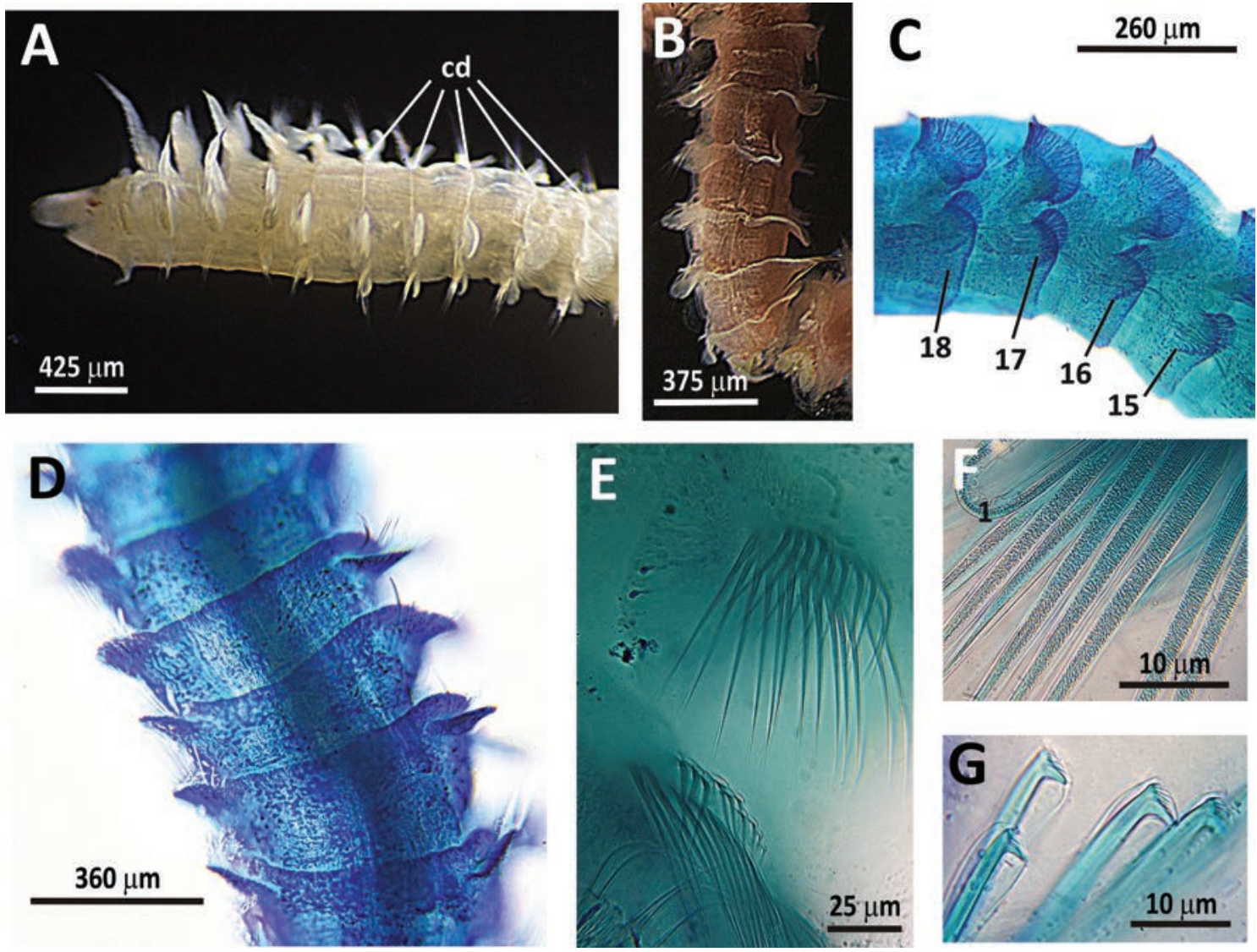

Fig. 5.- Prionospio rikardoi n. sp. A. Región anterior, vista dorsolateral (paratipo MNCN 16.01/18527). B. Setígeros 18-24, vista dorsal (paratipo MNCN 16.01/18533). C. Setígeros 15-18, vista lateral (paratipo MNCN 16.01/18527). D. Setígeros 13-18, vista ventral (paratipo MNCN 16.01/18528). E. Sedas capilares neuropodiales de los setígeros 7-8 (paratipo MNCN 16.01/18527). F. Detalle de las sedas capilares notopodiales del setígero 8 (paratipo MNCN 16.01/18527). G. Ganchos encapuchonados neuropodiales del setígero 23 (paratipo MNCN 16.01/18527). Abreviatura: cd: cresta dorsal.

Fig. 5.- Prionospio rikardoi n. sp. A. Anterior end, dorsolateral view (paratype MNCN 16.01/18527). B. Chaetigers 18-24, dorsal view (paratype MNCN 16.01/18533). C. Chaetigers 15-18, lateral view (paratype MNCN 16.01/18527). D. Chaetigers 13-18, ventral view (Paratype MNCN 16.01/18528). E. Neuropodial capillary chaetae of chaetigers 7-8 (paratype MNCN 16.01/18527). F. Detail of notopodial capillary chaetae of chaetiger 8 (paratype MNCN 16.01/18527). G. Neuropodial hooded hooks of chaetiger 23 (paratype MNCN 16.01/18527). Abbreviation: cd: dorsal crest.

rikardoi $\mathbf{n}$. sp., finas en $P$. fauchaldi), sedas neuropodiales del setígero 3 (de longitud similar a los otros setígeros en Prionospio rikardoi $\mathbf{n}$. sp., algunas extremadamente largas en P. fauchaldi), morfología de las crestas dorsales (con una solapa semicircular conspicua en el tramo central en P. laciniosa, sin ella en Prionospio rikardoi n. sp.) y forma de las lamelas neuropodiales del setígero 2 (cuadradas en $P$. vallensis, acuminadas y alargadas ventralmente en Prionospio rikardoi n. sp.).

\section{ECOLOGÍA}

En las costas del País Vasco, Prionospio rikardoi n. sp. habita en fondos circalitorales constituidos por arenas puras, arenas gruesas y gravas con moderados a bajos porcentajes de materia orgánica, a profundidades comprendidas entre 36 y $105 \mathrm{~m}$ de profundidad, en una biocenosis similar a la "Comunidad profunda de Venus" o a la "Asociación de gravas costeras boreal", descritas por Ford (1923) y Jones (1950). En estos fondos las especies con mayores abundancias han correspondido a los poliquetos Pisione remota (Southern, 1914), Sphaerosyllis bulbosa Southern, 1914, Goniadella gracilis (Verrill, 1873), Glycera lapidum Quatrefages, 1866, Protodorvillea kefersteini (McIntosh, 1869), Mediomastus fragilis Rasmussen, 1973, Polygordius appendiculatus Fraipont, 1887, los moluscos Limatula subauriculata (Montagu, 1808), Asbjornsenia pygmaea (Lovén, 1846) y Timoclea ovata (Pennant, 1777), el isópodo Eurydice truncata (Norman, 1868), tanaidáceos indeterminados de la familia Leptognathiidae, anfípodos indeterminados de la familia Bogidiellidae, la ofiura Amphipholis squamata (Delle Chiaje, 1828), el erizo irregular Echinocyamus pusillus (O.F. Müller, 1776), la holoturia Leptosynapta minuta (Becher, 1906) y nematodos indeterminados.

\section{DISTRIBUCIÓN}

Costas del País Vasco (Noroeste de la península Ibérica, sureste del golfo de Bizkaia). 
ETIMOLOGÍA

La especie está dedicada a Rikardo Adarraga, padre fallecido de Idoia Adarraga.

Clave de las especies de Prionospio con sólo branquias apinnadas comenzando en el segundo segmento setígero.

A partir de la clave elaborada por Dagli \& Çinar (2011) para el subgénero Minuspio y los recientes descubrimientos en fondos profundos del cañón Setúbal en Portugal (Paterson et al., 2016) y fondos someros y circalitorales de la península Ibérica (Delgado-Blas et al., 2019; presente estudio), la clave para todas las especies de Prionospio portadoras únicamente de branquias apinnadas y comienzo en el segundo setígero, quedaría actualizada de la siguiente manera:

1 Dos a cuatro pares de branquias presentes ................ 2

- Más de cuatro pares de branquias presentes .................. 10

2 Dos pares de branquias ........................................... 3

- Tres o cuatro pares de branquias................................ 4

3 Prostomio oval, anteriormente redondeado; carúncula extendiéndose hasta el margen anterior del setígero 1 ......Prionospio hermesia Neal \& Paterson in Paterson et al., 2016

- Prostomio rectangular; carúncula extendiéndose hasta el margen anterior del setígero 2

Prionospio kaplani Altamira, Glover \& Paterson in Paterson et al., 2016

4 Tres pares de branquias

Prionospio branchilucida Altamira, Glover \& Paterson in Paterson et al., 2016

- Cuatro pares de branquias .. 5

5 Algunas branquias con la superficie rugosa y/o sinuosa ............................................................... 6

- Todas las branquias con la superficie lisa ................ 8

6 Todas las branquias con la superficie rugosa y/o sinuosa; faldones laterales ausentes ........................ 7

- Primer y cuarto par de branquias con con la superficie rugosa y/o sinuosa; faldones laterales presentes.

Prionospio laciniosa Maciolek, 1985

7 Primer y cuarto par de branquias de similar longitud; sedas en sable relativamente finas.

Prionospio fauchaldi Maciolek, 1985

- Primer par de branquias de mayor longitud que el cuarto; sedas en sable robustas

Prionospio vallensis Neal \& Paterson in Paterson et al., 2016

8 Crestas dorsales presentes 9

- Crestas dorsales ausentes.

Prionospio japonicus Okuda, 1935

9 Branquias largas; lamela postsetal del neuropodio 2 redondeada....... Prionospio elegantula Imajima, 1990
- Branquias relativamente cortas; lamela postsetal del neuropodio 2 triangular, con el extremo ventral puntiagudo Prionospio rikardoi $\mathrm{n} . \mathrm{sp}$.

1039 pares de branquias

Prionospio polybranchiata Fauvel, 1929

- Menos de 39 pares de branquias ............................. 11

11 Bolsas interapodiales presentes

..............................Prionospio aluta Maciolek, 1985

- Bolsas interapodiales ausentes. 12

12 Sedas en sable ausentes

Prionospio perkensi Maciolek, 1985

- Sedas en sable presentes .... 13

13 Branquias claramente más largas (al menos 4-5 veces) que las lamelas postsetales notopodiales ............... 14

- Branquias relativamente cortas, similares o ligeramente más largas (1-1,5 veces) a las lamelas postsetales notopodiales 19

14 Prostomio con protuberancias marginales presentes...... 15 Prostomio sin protuberancias marginales ............... 18

15 Margen anterior del prostomio truncado con una pequeña protuberancia central

Prionospio delta Hartman, 1965

- Margen anterior del prostomio redondeado con 3-5 protuberancias marginales

16 Ojos ausentes

... Prionospio (Minuspio) anatolica Dagli \& Çinar, 2011

- Dos pares de ojos 17

17 Crestas dorsales ausentes

. Prionospio lighti Maciolek, 1985

Crestas dorsales presentes.

Prionospio pulchra Imajima, 1990

18 Branquias más largas en el setígero 2, prostomio más ancho en el tramo medio.

Prionospio tatura Wilson, 1990 Branquias más largas en los setígeros 4-5, prostomio más ancho en el tramo posterior...

Prionospio yuriel Wilson, 1990

19 Lamela postsetal del notopodio del setígero 1 ausente ...................................................... 20 Lamela postsetal del notopodio del setígero 1 presente ................................................................... 23

20 Crestas dorsales ausentes . Prionospio patagonica Augener, 1923

- Crestas dorsales presentes. 21

21 Sin protuberancias marginales en el prostomio ....Prionospio (Minuspio) maciolekae Dagli \& Çinar, 2011 Con protuberancias marginales. 22

22 Margen anterior del prostomio truncado y con sólo una protuberancia apical.

Prionospio wireni Maciolek, 1985 
- Margen anterior del prostomio redondeado con cinco protuberancia apicales

Prionospio multibranchiata Berkeley, 1927

23 Más de 10 pares de branquias..... 24

10 o menos pares de branquias 25

24 Crestas dorsales ausentes

- Crestas dorsales presentes

Prionospio wambiri Wilson, 1990

Prionospio elongata Imajima, 1990

25 Ojos ausentes, branquias fruncidas.

.. Prionospio sandersi Maciolek, 1981

- Uno o dos pares de ojos presentes; branquias lisas ........ 26

268 8-10 pares de branquias, sedas en sable a partir del setígero 12-16 .......... Prionospio (Minuspio) sanmartini

Delgado-Blas, Díaz-Díaz \& Viéitez, 2019

- 6-8 pares de branquias, sedas en sable a partir del setígero 10

Prionospio cirrifera Wirén, 1883

\section{Agradecimientos}

Los autores quieren agradecer al centro tecnológico AZTITECNALIA por la cesión del material aquí estudiado, así como al Departamento de Desarrollo Económico e Infraestructuras (Gobierno Vasco), Dirección de Biodiversidad, Departamento de Medio Ambiente, Planificación Territorial y Vivienda (Gobierno Vasco), Diputación Foral de Gipuzkoa, Aguas del Añarbe, Ente Vasco de la Energía (EVE), Biscay Energy Platform S.A. BIMEP (Grupo EVE) y URA por la financiación de las campañas oceanográficas en las que se obtuvieron los ejemplares de la nueva especie. Del mismo modo, expresamos nuestro agradecimiento al Dr. José Manuel Viéitez (Departamento de Ciencias de la Vida, Universidad de Alcalá de Henares, Madrid), así como a un revisor anónimo por sus comentarios y sugerencias que han contribuido a mejora la versión final del manuscrito.

\section{Referencias}

Blake, J. A., 1996. Family Spionidae Grube, 1850. In: Blake, J.A., Hilbig, B. \& Scott, P.H. (eds.). Taxonomic atlas of the benthic fauna of the Santa Maria Basin and Western Santa Barbara Channel, Vol. 6-the Annelida, part 3, Polychaeta: Orbiniidae to Cossuridae. Santa Barbara Museum of Natural History. Santa Barbara, CA: 81-223.

Blake, J. A. \& Kudenov, J. D., 1978. The Spionidae (Polychaeta) from southeastern Australia and adjacent areas with a revision of the genera. Memoirs of the Museum of Victoria, 39: 171-280.

Blake, J. A., Maciolek, N. J. \& Meißner, K., 2017. Spionidae, Grube 1850. In: Westheide, W. \& Purschke, G. (eds.), Handbook of Zoology. A Natural History of the Phyla of the Animal Kingdom. Annelida: Polychaetes. De Gruyter, Berlin. (published online).

Borja, A., Muxika, I. \& Franco, J., 2006. Long-term recovery of soft-bottom benthos following urban and industrial sewage treatment in the Nervión estuary (soutern Bay of Biscay). Marine Ecology Progress Series, 313: 43-55.
Dagli, E. \& Çinar, M. E., 2009. Species of the subgenera Aquilospio and Prionospio (Polychaeta: Spionidae: Prionospio) from the southern coast of Turkey (Levantine Sea, eastern Mediterranean), with description of a new species and two new reports for the Mediterranean fauna. Zootaxa, 2275: 1-20.

Dagli, E. \& Çinar, M. E., 2011. Species of the subgenus Minuspio (Polychaeta: Spionidae: Prionospio) from the southern coast of Turkey (Levantine Sea, eastern Mediterranean), with the description of two new species. Zootaxa, 3043: 35-53. https://doi.org/10.11646/zootaxa.3043.1.4

Delgado-Blas, V. H., Díaz-Díaz, O. \& Viéitez, J. M., 2019. Two new species of spionids from the genera Dispio and Prionospio (Polychaeta: Spionidae) from the Iberian Peninsula with redescription and notes on Prionospio (Minuspio) multibranchiata Berkeley, 1927. Zootaxa, 4604(3): 562-574. https://doi.org/10.11646/zootaxa.4604.3.11

Ford, E., 1923. Animal communities of the level sea-botom in the waters adjacent to Plymouth. Journal of the Marine Biological Association of United Kingdom, 13: 164-224. https://doi.org/10.1017/S0025315400010985

Holme, N. A. \& McIntyre, A. D., 1971. Methods for the study of marine benthos. Blackwell Scientific Publications. Oxford.

Imajima, M., 1990. Spionidae (Annelida, Polychaeta) from Japan. III. The genus Prionospio (Minuspio). Bulletin of the National Science Museum, Serie A, 16: 61-78.

Jones, N. S., 1950. Marine bottom communities. Biological Reviews of the Cambridge Philosophical Society, 25: 283-313.

Maciolek, N. J., 1985. A revision of the genus Prionospio Malmgren, with special emphasis on species from the Atlantic Ocean, and new records of species belonging to the genera Apoprionospio Foster and Paraprionospio Caullery (Polychaeta, Annelida, Spionidae). Zoological Journal of the Linnean Society, 84: 325-383. https://doi. org/10.1111/j.1096-3642.1985.tb01804.x

Mincks, S. L., Dyal, P. L., Paterson, G. L. J., Smith, C. R. \& Glover, A. G., 2009. A new species of Aurospio (Polychaeta, Spionidae) from the Antartic shelf, with analysis of its ecology, reproductive biology and evolutionary history. Marine Ecology, 30: 181-197. https://doi. org/10.1111/j.1439-0485.2008.00265.x

Okuda, S., 1935. Some lacustrine polychaetes with a list of brackish-water polychaetes found in Japan. Annotationes Zoologicae Japonenses, 15: 240-246.

Paterson, G. L. J., Neal, L., Altamira, I., Soto, E. H., Smith, C. R., Menot, L., Billet, D. S. M., Cunha, M. R., Marchais-Laguionie, C. \& Glover, A. G., 2016. New Prionospio and Aurospio Species from the Deep Sea (Annelida: Polychaeta). Zootaxa, 4092(1): 1-32.

Peixoto, A. J. M. \& De Paiva, P. C., 2019. New Prionospio and Laubieriellus (Annelida: Spionidae) species from Southeastern Brazil. Zootaxa, 4577(3): 529-547. https://doi.org/10.11646/zootaxa.4577.3.7

Radashevsky, V. I., 2015. Spionidae (Annelida) from Lizard Island, Great Barrier Reef, Australia: the genera Aonides, Dipolydora, Polydorella, Prionospio, Pseudopolydora, Rhynchospio, and Tripolydora. Zootaxa, 4019(1): 635-694. 
Read, G. \& Fauchald, K., 2019. World Polychaeta database. Prionospio Malmgren, 1967. Accessed through World Register of Marine Species at: http://www.marinespecies.org/aphia.php? $\mathrm{p}=$ taxdetails\&id=129620

Sigvaldadóttir, E., 1998. Cladistic analysis and classification of Prionospio and related genera (Polychaeta, Spionidae). Zoologica Scripta, 27: 175-187. https:// doi.org/10.1111/j.1463-6409.1998.tb00435.x

Sigvaldadóttir, E., 2002. Polychaetes of the genera Prionospio and Aurospio (Spionidae, Polychaeta) from
Icelandic waters. Sarsia, 87: 207-215. https://doi. org/10.1080/00364820260294842

Wilson, R. S., 1990. Prionospio and Paraprionospio (Polychaeta: Spionidae) from Southern Australia. Memoirs of Museum Victoria, 50: 243-274. https://doi. org/10.24199/j.mmv.1990.50.02

Zhou, J. \& Li, X. Z., 2009. Report of Prionospio complex (Annelida: Polychaeta: Spionidae) from China's waters, with description of a new species. Acta Oceanologica Sinica, 28: 116-127. 